\title{
Intussusception secondary to a Meckel's diverticulum in a 6-month-old male
}

\begin{abstract}
The differential diagnosis in a vomiting infant can be extensive, ranging from the benign viral gastroenteritis and gastro esophageal reflux to serious bowel pathologies such as intussusception and malrotation. Intussusception occurs when a proximal bowel segment becomes trapped in a distal bowel segment, most commonly in the ileocecal region, and can infrequently involve an anatomic lead point such as intestinal polyps, cancer, inflammatory patches, enteric duplication cysts, or a Meckel's diverticulum. We present a case of a 6 -month-old male admitted with presumed viral gastroenteritis who was found to have a necrotic Meckel's diverticulum associated intussusception requiring open laparotomy for correction. Clinicians should cultivate a high index of suspicion for intussusception in the vomiting child, as the diagnosis can be elusive and have serious consequences if unrecognized.
\end{abstract}

Volume 9 Issue I - 2019

\author{
Vanessa Rallis MD, Patrick Cazilas MD, Shane \\ Rainey DO \\ Department of Pediatrics, University of Illinois College of \\ Medicine at Peoria, USA
Correspondence: Shane Rainey, Department of Pediatrics, University of Illinois College of Medicine at Peoria, OSF Healthcare Children's Hospital of Illinois, 530 NE Glen Oak Ave., Peoria, IL, 61637, Tel (817) 706-207I,
Email shane.c.rainey@osfhealthcare.org

Received: January 26, 2019 | Published: February II, 2019

\section{Introduction}

Intussusception is the most common bowel emergency and most common cause of intestinal obstruction in early childhood. It is caused by an invagination of a proximal intestinal segment into its distal counterpart, most commonly at the ileocecal junction. While commonly thought to be associated with a pathological lead point, the majority of cases are idiopathic, and a lead point is only identified in approximately 25 percent of pediatric cases. ${ }^{1}$ We present a case of an undiagnosed intussusception secondary to a necrotic Meckel's diverticulum masquerading as viral gastroenteritis in a 6-month-old infant. He failed air enema reduction and required open laparotomy and $15 \mathrm{~cm}$ of bowel resection for correction. Clinicians should be mindful of intussusception when developing their differential diagnosis for the child with vomiting and abdominal pain.

\section{Clinical presentation}

A 6-month-old ex-33 week preemie infant was seen in a referring emergency department (ED) for one day of vomiting and poor oral intake. His mother reported that the day prior to presentation, he had four episodes of non-bloody, non-bilious emesis and had subjectively decreased urine output. His last bowel movement was the day prior to presentation and was soft and of usual caliber according to his mother. There were no reported fevers, diarrhea, rashes, household sick contacts, and daycare exposure. He has previously been in his usual state of health and has no chronic medical conditions. He was born at 33 weeks after his mother presented in preterm labor and was admitted to the neonatal intensive care unit (NICU) for two weeks. Other than requiring supplemental oxygen for several days, his stay in the NICU was unremarkable. At the referring ED, his complete blood count (CBC), basic metabolic profile (BMP), c-reactive protein, and urinalysis were within normal limits. An abdominal radiograph was read as having a moderate stool burden. Urine and blood cultures were obtained prior to a dose of ceftriaxone, and he was admitted to their inpatient unit where an orogastric (OG) tube was placed to attempt rehydration. The infant continued vomiting despite OG rehydration and was transferred to our institution after several attempts at intravenous (IV) access were unsuccessful. Physical examination upon arrival was notable for a slightly sunken anterior fontanel, dry mucous membranes, capillary refill of 4 seconds, and a soft, non-distended abdomen without hepatosplenomegaly. A repeat $\mathrm{CBC}$ and BMP were unremarkable, an IV was successfully placed for fluid resuscitation, and he was made nil per os (NPO) for bowel rest for a presumed diagnosis of viral gastritis. On hospital day two, the infant developed abdominal distension thought to be secondary to a high stool burden and he was given a glycerin suppository. Shortly thereafter, the patient had an episode of hematochezia which had the appearance of currant jelly. An urgent abdominal radiograph showed dilated loops of bowel in the mid abdomen suggestive of a small bowel obstruction (Figure 1). A nasogastric (NG) tube was placed for decompression and an abdominal ultrasound was performed which revealed a "target sign" in the right lower abdomen suggestive of an ileocecal intussusception (Figure 2). Surgery and radiology were consulted for further evaluation and, after two air enemas were unsuccessful in reducing the intussusception, surgery took the patient to the operating room. Laparoscopic reduction was also unsuccessful, and the case was converted to an open laparotomy. Upon surgical examination, approximately $15 \mathrm{~cm}$ of strangulated, non-viable small bowel was noted extruding into the ascending colon, preceded by a necrotic Meckel's diverticulum which was thought to be the pathologic lead point. The necrotic bowel and diverticulum were resected, followed by anastomosis of the viable bowel segments. Over the next three days, the infant's bowel function recovered, the NG tube was removed, he was able to tolerate oral feedings, and was subsequently discharged.

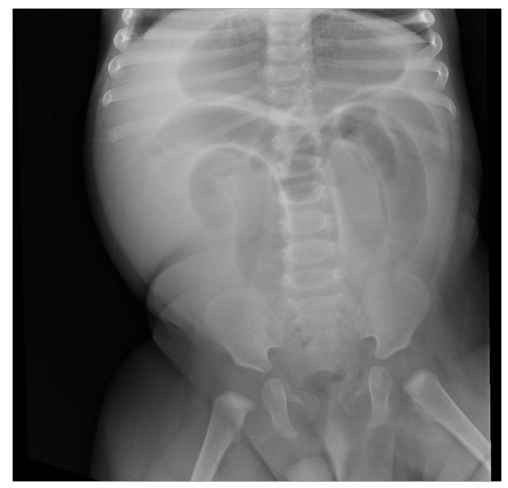

Figure I Abdominal radiograph demonstrating multiple dilated loops of bowel in the middle abdomen consistent with a small bowel obstruction. 


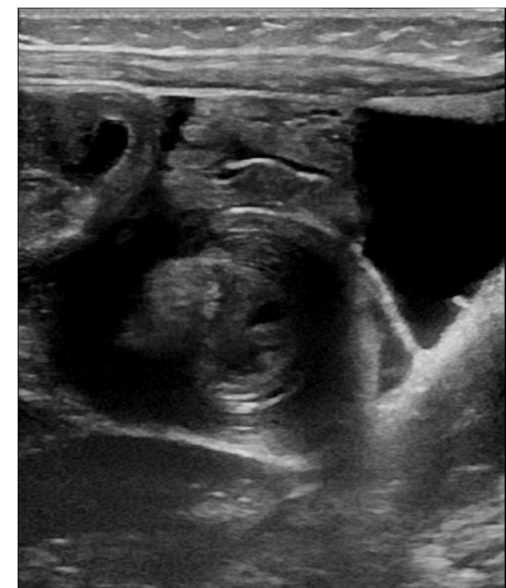

Figure 2 Ultrasound of the right lower abdomen demonstrating ileocolic intussusception.

\section{Discussion}

The vomiting infant can present the clinician with a diagnostic challenge, as the differential diagnosis is broad. Potential diagnoses include reflux, gastroenteritis, pyloric stenosis, small bowel obstruction, intussusception, and malrotation, among others. In many cases of uncomplicated gastritis or gastroenteritis, the vomiting is self-limited and usually does not require further investigation or intervention in an otherwise healthy patient with a normal physical exam. Thus, clinicians must maintain a low threshold to broaden the differential diagnosis in circumstances where a patient fails to improve or worsens despite the appropriate management. In the present case, the new onset abdominal distension and subsequent hematochezia is suggestive of a more ominous intra-abdominal process such as intestinal ischemia or bowel obstruction and warrants further inquiry to determine the true etiology of the patient's symptoms. Intussusception is the most common bowel emergency and most common cause of intestinal obstruction in children 3 to 36 months of age. ${ }^{2}$ The condition occurs when a proximal segment of bowel is pulled antegrade and becomes trapped in a distal segment of bowel. More than $90 \%$ of cases involve the ileocecal region, when the ileum enters and becomes trapped in the ascending colon.

Most cases are idiopathic as there is no clear triggering disease or pathologic lead point. Many of these idiopathic occurrences are thought to be associated with viral intestinal infections, such as rotavirus and adenovirus. ${ }^{3,4} \mathrm{~A}$ lead point is identified in approximately 25 percent of cases and can be secondary to a variety of conditions including intestinal polyps, vascular malformation, lymphoma, immunoglobulin A vasculitis, duplication cysts, and Meckel's diverticula. ${ }^{1}$ Initial presenting symptoms are often nonspecific and can include abdominal pain, vomiting, dehydration, and lethargy. The classic presentation of an intussusception involves severe sudden onset abdominal pain, during which the child will bring the legs towards the abdomen. If the intussusception is sustained, it may present with signs of obstruction, such as abdominal distension, bilious emesis, and hematochezia indicating vascular compromise.

The classically described currant jelly stool containing blood and mucous is seen infrequently. Occasionally, a sausage shaped mass may be palpated in the abdomen on physical exam. Overall, the classic triad of acute onset abdominal pain, currant jelly stool, and a palpable sausage shaped mass is seen in less than 15 percent of cases, making the diagnosis frequently elusive. ${ }^{5}$ Ultrasound remains the standard initial modality in the evaluation of a child with suspected intussusception and has a sensitivity and specificity of nearly 100 percent when performed by an experienced ultrasonographer. ${ }^{6,7}$ Ultrasound classically reveals the path gnomonic "target sign," representing bowel within bowel. A plain abdominal radiograph is helpful to exclude bowel perforation, which would require immediate surgical intervention, but may also reveal signs of intestinal obstruction. ${ }^{8,9}$ Air or contrast enema is the initial therapeutic modality of choice, and is successful in reducing the intussusception in 83 and 70 percent of cases, respectively. ${ }^{10,11}$ Surgical management is indicated if the patient is unstable, there is intestinal perforation, or non-operative reduction is unsuccessful.

\section{Conclusion}

The vomiting infant can present the clinician with a diagnostic challenge, as serious diagnoses may masquerade as commonly benign conditions. Intussusception is the most common cause of bowel obstruction in childhood and requires a high index of suspicion to diagnose. Given the possibility of progression to serious illness, clinicians should be mindful of intussusception when developing their differential diagnosis for the child with vomiting and abdominal pain.

\section{Funding source}

No funding was secured for this study.

\section{Financial disclosure}

The authors have no financial relationships relevant to this article to disclose.

\section{Acknowledgments}

None.

\section{Conflicts of interest}

The authors have no conflicts of interest to disclose.

\section{References}

1. Ntoulia A, Tharakan SJ, Reid JR, et al. Failed intussusception reduction in children: correlation between radiologic, surgical, and pathologic findings. AJR Am J Roentgenol. 2016;207(2):424-433.

2. Mandeville K, Chien M, Willyerd FA, et al. Intussusception: clinical presentations and imaging characteristics. Pediatr Emerg Care. 2012;28(9):842.

3. Bhisitkul DM, Todd KM, Listernick R. Adenovirus infection and childhood intussusception. Am J Dis Child. 1992;146(11):1331-1333.

4. Montgomery EA, Popek EJ. Intussusception, adenovirus, and children: a brief reaffirmation. Hum Pathol. 1994;25(2):169.

5. Losek JD, Fiete RL. Intussusception and the diagnostic value of testing stool for occult blood. Am J Emerg Med. 1991;9(1):1-3.

6. Ko HS, Schenk JP, Tröger J, et al. Current radiological management of intussusception in children. Eur Radiol 2007;17(9):411-21.

7. Hryhorczuk AL, Strouse PJ. Validation of US as a first-line diagnostic test for assessment of pediatric ileocolic intussusception. Pediatr Radiol. 2009;39(10):1075-1079.

8. Morrison J, Lucas N, Gravel J. The role of abdominal radiography in the diagnosis of intussusception when interpreted by pediatric emergency physicians. J Pediatr 2009;155(4):556-559.

9. Saverino BP, Lava C, Lowe LH, et al. Radiographic findings in the diagnosis of pediatric ileocolic intussusception: comparison to a control population. Pediatr Emerg Care. 2010;26(4):281-284. 
10. Beres AL, Baird R. An institutional analysis and systematic review with meta-analysis of pneumatic versus hydrostatic reduction for pediatric intussusception. Surgery. 2013;154(2):328-334.
11. Sadigh G, Zou KH, Razavi SA, et al. Meta-analysis of Air Versus Liquid Enema for Intussusception Reduction in Children. AJR Am J Roentgenol. 2015;205(5):W542-W549. 